\title{
A NEW PARADIGM IN FAST BCD DIVISION USING ANCIENT INDIAN VEDIC MATHEMATICS SUTRAS
}

\author{
Diganta Sengupta ${ }^{1}$, Mahamuda Sultana ${ }^{2}$, Atal Chaudhuri ${ }^{3}$ \\ ${ }^{1}$ Future Institute of Engineering and Management, Kolkata, West Bengal, India \\ sg.diganta@gmail.com \\ ${ }^{2}$ Swami Vivekananda Institute of Science and Technology, \\ Kolkata, West Bengal, India \\ sg.mahamuda@gmail.com \\ ${ }^{3}$ JadavpurUniversity, Kolkata, West Bengal, India \\ atalc23@gmail.com
}

\begin{abstract}
For decades, division is the most time consuming and expensive procedure in the Arithmetic and Logic Unit of the processors. This paper proposes a novel division algorithm based on Ancient Indian Vedic Mathematics Sutras which is much faster compared to conventional division algorithms. Also large value for the dividend and the divisor do not adversely affect the speed as time estimation of the algorithm depends on the number of normalizations of the remainder rather than on the number of bits in the dividend and the divisor. The algorithm has exhibited remarkable results for conventional midrange processors with numbers of size around 50 bits(15 digit numbers), the upper ceiling of computable numbers for conventional algorithms and the algorithm can divide numbers having size up to 38 digits (127 bits) with conventional processor in the present form, if modified it can divide even bigger numbers.
\end{abstract}

\section{KEYWORDS}

Division Algorithm, Fast BCD Division, Vedic Division Algorithm

\section{INTRODUCTION}

The Ancient Indian Vedic Mathematics comprises of sixteen Sutras and thirteen corollaries [1-2]. The four elementary operations of a processor, the addition, subtraction, multiplication and the division have been extensively dealt with in the sixteen sutras of Vedic Mathematics. The work in this paper involvesthe Nikhilam and the Paravartya Sutrawhich deal with the division. The Nikhilam Sutra also deals with multiplication andsome amount of work has been doneusing another sutra known as the UrdhvaTiryakbhyam Sutra [3-8]. The novelty of the Vedic Division Algorithm lies in the fact that the procedure incorporates addition and negation operations, both of which are much faster than the traditional successive subtraction methods. The Nikhilam Sutra can be stated as follows:

\subsection{The Nikhilam Sutra}

1. This Sutra breaks up the dividendinto two parts, one part resembling the Quotient and the other part resembling the Remainder. The number of digits in the Remainder part equals the number of digits in the divisor. For example,if the dividend and divisor are 2002002 and 89998 respectively, then 2002002 is broken up into two parts - 20 (part 1) and 02002 (part 2).

David C. Wyld (Eds) : ICCSEA, SPPR, CSIA, WimoA - 2013 
2. The next step in the Sutraadjusts the divisor by complimentingitusing the procedure "subtract all from 9 and the last from 10" in which all the digits in the divisor are subtracted from 9 barring the last significant digit which is subtracted from 10.Therefore, the divisor 89998 after adjustment becomes 10002 .

3. Next, the first digit of the quotient part (part 1 of the dividend) is divided by the first digit of the actual divisor. This is the only step where division is unavoidable, but in this case also the maximum division is that of a single digit number by a single digit number. In our work a look-up table has been created which stores all the single digit division results in the form of quotient and remainder, and accessed on demand.The first digit of the quotient part ' 2 'is divided by ' 8 ' (the first digit of the actual divisor) and the remainder ' 2 ' is noted down.

4. In this step, the remainder from the previous step is first written as the most significant digit of the quotient part and then it is multiplied by the adjusted divisor and placed below the dividend after shifting it one place right. This multiplication can be achieved by either the Nikhilam Sutra or the UrdhvaTiryakbhyam Sutra.

$\begin{array}{lll}89998 \quad & 2002002 \\ 10002 \quad & 20 / 02002 \\ & 2 / 0004 \\ & 2 \underline{22} / \ldots \ldots \ldots . . .\end{array} \quad$ : Divisor adjustment

5. In the above example, we observe that after the single digit multiplication, the digits in the quotient part (part 1) of the dividend have been added. Now the adjusted divisor is again multiplied by the new digit (marked by bold and underlined in example of step-4) to obtain another number and place it again by shifting it to the right by one position as shown below in Example-1:

\begin{tabular}{cccc}
89998 & ) & 2002002 & \\
10002 & $20 / 02002 \quad$ : Divisor adjustment \\
& $2 / 0004 \quad$ : Result after Step 4 \\
& \multicolumn{1}{c}{$/ 20004 \quad$ : Result after Step 5 } \\
\cline { 2 - 2 } & \multicolumn{2}{c}{$22 / 22046$}
\end{tabular}

Example 1.

Here it can be seen that since the result after step 5 has entered fully into the remainder part (part 2 ); hence the algorithm is concluded by adding all the intermediate results. Therefore, after dividing 2002002 by 89998 , we get the quotient as 22 and the remainder as 22046 .

\section{Observations}

It can be concluded that no subtraction procedure is performed in the entire division process.In the third step of the sutra, a single digit division has been done but that can be performed using a look-up table.The division process has been performed with multiplication process in subsequent steps and addition. Multiplication is a relatively faster and cheaper operation than division. Also the largest multiplication that may be required is multiplying 9 by 9 . 


\section{Drawback of the Nikhilam Sutra}

The sutra provides best results when division requires large divisors. In cases where the divisor is a small number, this sutra provides ambiguous results. This drawback is accomplished by another sutra known as the Paravartya Sutra.

\subsection{The Paravartya Sutra}

The Paravartya Sutra is suitable for divisions including large as well as small divisors. The sutra is actually known as "ParavartyaYojayet" which means "Transpose and Apply". The Paravartya Sutra can be easily explained using the famous Remainder Theorem [9] as follows:

1. If $\mathrm{E}=$ Dividend, $\mathrm{D}=$ Divisor, $\mathrm{Q}=\mathrm{Quotient}$ and $\mathrm{R}=$ Remainder and if the divisor is taken to be $(x-p)$, then a relationship can be stated as follows:

$$
\mathrm{E}=\mathrm{D} . \mathrm{Q}+\mathrm{R} \text {, or } \quad \mathrm{E}=\mathrm{Q} .(\mathrm{x}-\mathrm{p})+\mathrm{R} \text {. }
$$

2. Now, if ' $\mathrm{x}$ ' is substituted by ' $\mathrm{p}$ ' then the identity becomes $\mathrm{E}=\mathrm{R}$, thus the expression $\mathrm{E}$ automatically becomes the remainder as 'p' is achieved by equating $\mathrm{x}-\mathrm{p}$ to zero. Hence, actually the sign of ' $p$ ' is reversed [1].

3. In Paravartya Sutrathe digits of the divisor are first negated, i.e. if the divisor is 112 , the new adjusted divisor becomes $-1-1-2$. Then the first digit is excluded and the remaining digits become the new divisor $-1-2$.

4. Next the dividend part is broken up into two parts as in the Nikhilam Sutra and the operation is processed as shown in the Example-2.

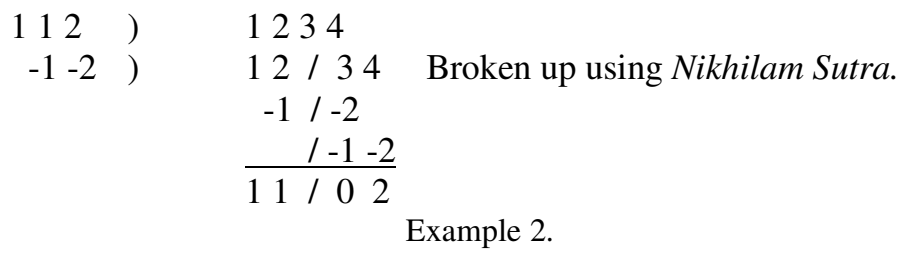

On close observation it can be seen that the first digit of the divisor,which was excluded initially,actually divides the first digit of the dividend as in the case of the Nikhilam Sutra, and the remainder obtained from that single digit division is used to multiply the adjusted divisor in the Paravartya Sutraand then proceeded with the Nikhilam Sutra to provide the quotient as 11 and the remainder as 2 .

\section{PROPOSED DIVISION ALgORITHM}

The proposed division algorithm in this paper is a combination of the earlier discussed two sutras, the Nikhilam Sutra and the Paravartya Sutra, with slight modification so as to obtain a generalized algorithm for all the possible divisors.

Presently, numerous division algorithms are used depending upon system or application requirements such as theRestore Type Division algorithm, SRT Division Algorithm, and the Non Restore Type Division Algorithm [10-12], the latter being the fastest and economic. It has been statistically proven further in our work that the proposed algorithm performs better with respect to the Non Restore Type Division Algorithmin terms of speed and memory requirement.

The proposed algorithm performs the calculations on the number of digits in the divisor and the dividend rather than on the number of bits representing them. In Non Restore Type Division Algorithm, the time estimate of the division is proportional to the number of bits. But in the Vedic 
Division Algorithm, the time requirement is based mainly on the number of normalizations (illustrated further) of the intermediate remainders. Hence, the algorithm exhibits remarkable results on divisions involving big numbers. The novelty of the algorithm lies in the fact that since the computation is done on digits rather than on the bits, very large numbers, having size up to 38 digits (127 bits), can be divided in the present form and if modified, it can divide even larger numbers.

\subsection{Step - by - Step Algorithm description using an example}

1. In the first step the divisor is adjustedusing a combined logic of both the Nikhilam Sutra and the Paravartya Sutra. All the digits that are less than or equal to 5 are negated. For all those digits which have values more than 5, 10's compliment of the digit is taken and 1 is added to the next higher digit. If the divisor is 47483647 , then a close observation reveals that all the consecutive digits are alternately less than and greater than 5 . The divisor adjustment starts from the Least Significant Digit. As $7>5$, hence 10's compliment of 7 is taken and 1 is added to the next higher digit ' 4 ', replacing 7 by 3 and 4 by 5 . Now this 5 is adjusted by -5 . Hence the adjustment of the divisor is shown below.

$$
\begin{array}{rrrrrrrrl}
4 & 7 & 4 & 8 & 3 & 6 & 4 & 7 & \text { becomes } \\
-5 & 3 & -5 & 2 & -4 & 4 & -5 & 3 & \text { (Adjusted divisor) }
\end{array}
$$

Let us take an example in which the dividend is 99999 and the divisor is 456 . Therefore, the divisor 456 after adjustment becomes -544 . It may also be observed that the first digit of an adjusted divisor will always be a negative digit.

2. Next, the first digit of the dividend is divided by the magnitude of the first digit of the adjusted divisor to obtain the quotient. In the example, the first digit of the dividend (99999) 9 is divided by magnitude of the first digit of the adjusted divisor (-5 4 4) 5 to obtain the quotient 1 . The new quotient is then multiplied by all the digits of the adjusted divisor and placed below the dividend at the exact positions and then added to get the new remainder as shown below:

$$
\begin{aligned}
& \begin{array}{rllllll}
-544 & 9 & 9 & 9 & 9 & 9 & (10000
\end{array} \\
& \text { ) } \begin{array}{rrrr}
-5 & 4 & 4 & \\
\hline 13 & 13 \quad 9 \quad 9
\end{array}
\end{aligned}
$$

3. This step normalizes the remainder. Normalization means replacinga multiple digit value by a single digit value at each position. The procedure is started from the Least Significant Number of the remainder and followed to the Most Significant Number. The Least Significant Digit of the multiple digit number is kept at the position and the rest is added to the next Higher Significant Digit. The procedure is shown below:

$\begin{array}{rrrrrl}4 & 13 & 13 & 9 & 9 & \\ 4 & 14 & 3 & 9 & 9 & \\ 5 & 4 & 3 & 9 & 9 & \text { : Normalized Remainder }\end{array}$

4. The next step checks for a ' 0 ' at the Most Significant Digit of the normalized remainder. If it is not ' 0 ', as in this case, then the normalized remainder is again divided by the adjusted divisor and the new quotient is added to the previous quotient. Else if it is ' 0 ', then the previous procedures are repeated till completion. The whole procedure is shown in the output snippet in Fig.1 below: 


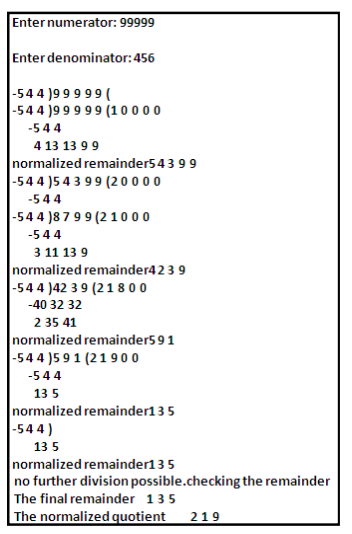

Fig.1.

It can be seen that the remainder normalization forms the main time consuming part of the algorithm. Also there is no standard procedure for predicting the number of normalizations. Hence, the time estimate does not depend on the size of the number. Even in divisions having higher number of normalizations, Vedic Division Algorithm performs better with respect to the conventional algorithms.

Statistically it has been found that in division intensive environments, like cryptographic algorithms etc, the overall performance of the Vedic Division Algorithm is much faster when compared to the conventional Non Restore Type Division Algorithm.

\subsection{The Algorithm}

\section{Initialization Part.}

1. The dividend and the divisor are held in two arrays - Array ' $a$ ' and Array ' $b$ ' having index ' $m$ ' and ' $n$ ' respectively. Array ' $b$ ' finally stores the remainder. A temporary array 'temp' has been used to hold the quotient havingindex ' $t$ '. Another array ' $c$ ' is used to hold the copy of the divisor. All the data has been stored in Little Endean Formats and initialized to 0.The length of Array ' $b$ ' is ' $n$ ' and Array ' $a$ ' is ' $m+1$ ', Array ' $c$ ' is ' $m$ ' and 'temp'is ' $n$ ' respectively.

2. The divisor is adjusted so that no digit in the divisor is more than 5 .

3. ' $\mathrm{m}$ ' is updated if adjusting the divisor causes increase in length of the divisor.

4. Inverse each digit of the divisor (including the most significant digit). Steps 3 and 4 have been clubbed in Article 2.1.

\section{Division Part.}

5. A new variable ' $\mathrm{j}$ ' is taken for holding the value of the possible number of iterations and its limits are set from 1 to ' $n-m+1$ '.

6. Compare the most significant digits of divisor and dividend.

7. If MSD (Most Significant Digit) of divisor $>$ MSD of dividend, club the most significant pair of digits of dividend. Update (decrement) $n$ and $t$.

8. If clubbing results in decrease of the number of digits of dividend below that of divisor, break from the 'For Loop using ' $\mathrm{j}$ '” and go to step 13.

9. Quo= MSD of dividend/ MSD of divisor.Calculate the remainder (dividend).

10. If MSD of dividend not divisible by that of divisor, then break from the "for loop". A fresh iteration is being started. Go to step 13 .

11. Decrement $\mathrm{n}$ and $\mathrm{t}$, go to step 8 . 
12. The remainder is then normalized.Increment ' $n$ ' and ' $t$ ' if normalization increments the number of digits in remainder.

13. If $(n<m)$ or if $(j>n-m+1)$ in the previous iteration, no further iterations are possible and $2^{\text {nd }}$ condition means we have already tried to divide once more but failed, go to step 16.

14. Else division is possible, go to step 7 .

15. Check the remainder. If the remainder is negative, the quotient is decremented once. Else if it is positive, it is the 'normalized version of divisor' and cannot be divided again. So check if original form of divisor (stored in array ' $c$ ') can divide again. This division can result into incrementing the quotient by 1 .

16. The quotient is stored in array 'temp' with the leading and trailing 0'sand remainder in array ' $b$ '. Quotient can further be normalized.

\subsection{The Flowchart}

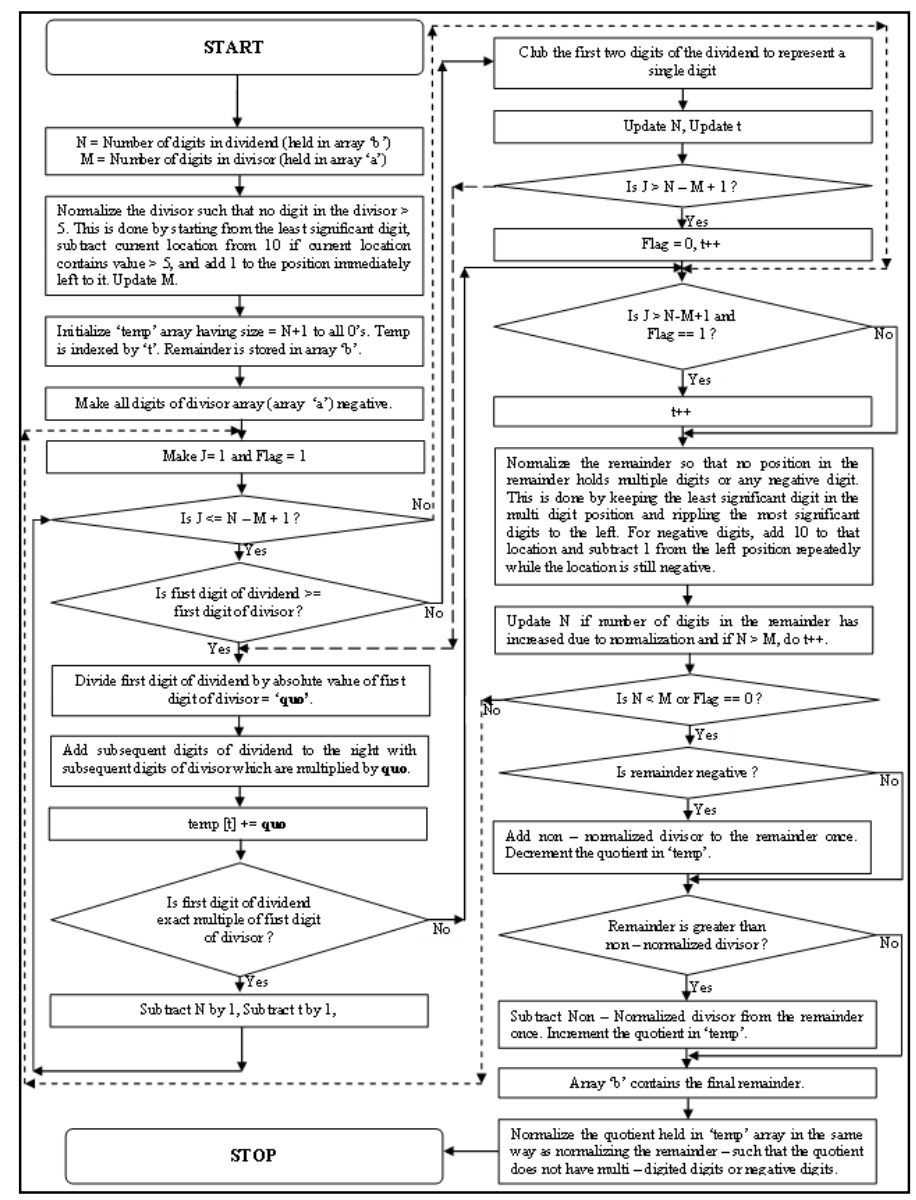

Fig.2.

\subsection{Division examples illustrating the Best Case and The Worst Case}

\section{Best Case.}

Let the dividend be 80217727 and the divisor be 20202. The output snippet of the division is shown in Fig. 3. It can be observed that remainder is normalized only in the last step. Hence, this type of division can be termed as a Best Case. 


\section{Worst Case.}

The example shown in Fig.1,Article 2.1, can be said to be a Worst Case as in almost all the steps, remainder normalization has been required.

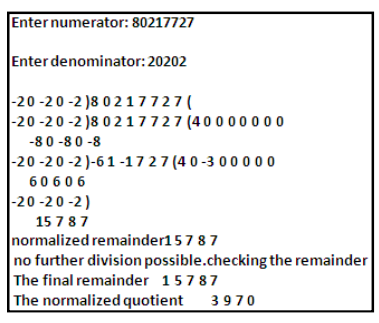

Fig.3.

\section{Performance Analysis of The Vedic Division Algorithm}

The analysis details were noted down as shown in Table 1 .

\begin{tabular}{|c|c|c|c|c|}
\hline \multirow{2}{*}{ Dividend } & \multirow{2}{*}{$\begin{array}{l}\mathbf{D} \\
\mathbf{i} \\
\mathbf{g} \\
\mathbf{i} \\
\mathbf{t} \\
\mathbf{s}\end{array}$} & \multirow{2}{*}{ Divisor } & $\begin{array}{c}\text { Vedic } \\
\text { Division }\end{array}$ & $\begin{array}{c}\text { Non } \\
\text { Restore } \\
\text { Division } \\
\end{array}$ \\
\hline & & & $\begin{array}{c}\text { Time in } \\
\mu \mathrm{s}\end{array}$ & Time in $\mu \mathrm{s}$ \\
\hline 91 & 2 & 16 & 0.150 & 0.800 \\
\hline 255 & 3 & 127 & 0.150 & 1.710 \\
\hline 948 & 3 & 182 & 0.310 & 2.340 \\
\hline 1,982 & 4 & 27 & 0.310 & 2.810 \\
\hline 3,728 & 4 & 94 & 0.160 & 3.270 \\
\hline 47,386 & 5 & 28 & 0.620 & 5.610 \\
\hline 131,071 & 6 & 7,295 & 0.460 & 5.780 \\
\hline 461,938 & 6 & 682 & 0.930 & 7.020 \\
\hline $1,048,576$ & 7 & 2,249 & 0.460 & 8.260 \\
\hline $3,729,618$ & 7 & 30,901 & 0.320 & 9.200 \\
\hline $10,388,608$ & 8 & 240 & 0.630 & 10.780 \\
\hline $80,217,727$ & 8 & 20,202 & 0.310 & 13.570 \\
\hline $482,937,164$ & 9 & 456 & 1.250 & 15.740 \\
\hline $736,582,914$ & 9 & 1,782 & 1.240 & 17.150 \\
\hline 1073741824 & $\begin{array}{l}1 \\
0\end{array}$ & 262125 & 1.860 & 16.860 \\
\hline 4294967295 & $\begin{array}{l}1 \\
0 \\
\end{array}$ & $\begin{array}{r}214748364 \\
7 \\
\end{array}$ & 0.610 & 17.770 \\
\hline 19372864582 & $\begin{array}{l}1 \\
1 \\
\end{array}$ & 4286 & 0.930 & 26.350 \\
\hline 965274638525 & $\begin{array}{l}1 \\
2 \\
\end{array}$ & 8258 & 1.240 & 31.050 \\
\hline 9561346784625 & $\begin{array}{l}1 \\
3 \\
\end{array}$ & 764318 & 2.780 & 39.910 \\
\hline 45615935785265 & $\begin{array}{l}1 \\
4 \\
\end{array}$ & 646464 & 3.120 & 40.080 \\
\hline 56455825519553 & $\begin{array}{l}1 \\
4 \\
\end{array}$ & 61945 & 3.900 & 41.640 \\
\hline 693582471951753 & $\begin{array}{l}1 \\
5 \\
\end{array}$ & 84265 & 2.490 & 48.760 \\
\hline 731984265735195 & $\begin{array}{l}1 \\
5\end{array}$ & 289357 & 2.490 & 49.290 \\
\hline
\end{tabular}


For performance analysis, the Vedic Division Algorithm was executed on a 32 bit Operating System having Intel Pentium Dual CPU E2180 @2.00 GHz and 0.99 GB DDR2 RAM. At each execution, the algorithm was iterated 100,000 timeswith the same dividend and the divisor and the average time was noted, as the time taken for a single execution was so minuscule that it could not be detected. We think that it is the most rational method for time estimation. The comparison was made with respect to the Non Restore Type Division Algorithm, which was also iterated for an equal number of times for the same set of dividends and divisors, and the average execution time for this algorithm was also noted. Each set of dividends and divisors ware executed by both the algorithms for seven times and the minimum value of time required of the seven executions were taken for analysis. This was done due to factors influencing the time analysis of the execution such as operating system time scheduling. The dividends and the divisors were taken in a random manner and the results were tabulated. It can be seen that in the last row in Table 1, the dividend is a 15 digit number or in terms of bits, it is a 50 bit number. In this range the Non Restore Type Division Algorithm starts giving ambiguous results but the Vedic Division Algorithm performs satisfactorily. Also the Vedic Division Algorithm can compute numbers having 38 digits, 127 bit numbers, accurately in the present form, if modified it can divide even larger numbers. The tabulated data in Columns 4 and 5 in Table 1 have been analyzed further in Fig. 4.

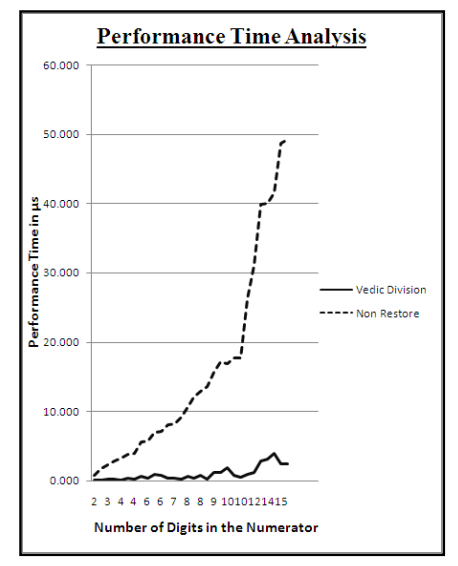

Fig.4.

It can be well observed in Fig.4 that with increase in value in the dividend, the performance time in case of Non Restore Type Division Algorithm increases drastically compared to the Vedic Division Algorithm. This is because the Non Restore Type Division Algorithmcomputes on the number of bits, hence with increasing number of bits, the execution time increases. It can also be seen that there are a few glitches while computing with the large sized numbers in the Vedic Division Algorithm, but those are due to the random selection of dividends and the divisors, otherwise it can be stated that the computation time required by the Vedic Division Algorithm is almost constant irrespective of the size of the dividend.

\section{CONCLUSION}

The Vedic Division Algorithm has exhibited remarkable results with respect to conventional division algorithms in terms of fast BCD division. Also it has been observed that the execution time does not depend on the size of the dividend or the divisor, but on the number of remainder normalizations required. Further VLSI implementation of the algorithm remains to be tested. In terms of Fast BCD Division, it has again been proven by Ancient Indian Vedic Mathematics that 'Old is Gold'. 


\section{REFERENCES}

[1] Jagadguru Swami Sri Bharath, KrsnaTirathji, "Vedic Mathematics or Sixteen Simple Sutras From The Vedas", MotilalBanarsidas , Varanasi(India),1986.

[2] Swami Bharati Krishna Tirtha's Vedic mathematics [Online]. Available: http://en.wikipedia.org/wiki/Vedic mathematics.

[3] HimanshuThapliyal, R.V Kamala and M.B Srinivas "RSA Encryption/Decryption in Wireless Networks Using an Efficient High Speed Multiplier", Proceedings of IEEE International Conference On Personal Wireless Communications (ICPWC-2005), New Delhi, pp-417-420, Jan 2005.

[4] HimanshuThapliyal and M.B Srinivas, "High Speed Efficient Hierarchical Overlay Multiplier Architecture Based on Ancient Indian Vedic Mathematics", Proceedings of International Conference on Signal Processing, ICSP 2004, Turkey, Dec 2004.

[5] HimanshuThapliyal and M.B Srinivas, A High Speed and Efficient Method of Elliptic CurveEncryption Using Ancient Indian Vedic Mathematics.

[6] ManoranjanPradhan, Rutuparna Panda and Sushanta Kumar Sahu, "Speed Comparison of 16x16 Vedic Multipliers”, International Journal of Computer Applications (0975 - 8887), Volume 21- No.6, May 2011

[7] Harpreet S. Dhillon and A.Mitra , “A Digital Multiplier Architecture using UrdhvaTiryakbhyam Sutra of Vedic Mathematics “,Department of Electronics and Communication Engineering,Indian Institute of Technology, Guwahati 781 039, India.

[8] Purushottam D. ChidgupkarMangesh T. Karad, The Implementation of Vedic Algorithms in DigitalSignal Processing, Global J. of Engg. Educ., Vol.8, No.2 () 2004 UICEE, Published in Australia.

[9] Shuangching Chen and ShugangWei,"A High-Speed Realization of Chinese Remainder Theorem”, Proceedings of the 2007 WSEAS Int. Conference on Circuits, Systems, Signal and Telecommunications, Gold Coast, Australia, January 17-19, 2007

[10] Stuart F. Oberman, and Michael J. Flynn, "Division Algorithms and Implementations", IEEE Transactions on Computers, vol. 46, no. 8, August 1997.

[11] J. O'Leary, M. Leeser, J. Hickey and M. Aagaard, "Non-Restoring Integer Square Root: A Case Study in Design by Principled Optimization", LNCS.

[12] Website: csclab.murraystate.edu/bob.pilgrim/405/Computing\%20Machinery\%20Ch06.pdf. 